\title{
Variability of intraoperative electrostimulation parameters in conscious individuals: language cortex
}

\author{
Franck-Emmanuel Roux, MD, PhD, ${ }^{1-3}$ Jean-Baptiste Durand, PhD, ${ }^{3}$ Imène Djidjeli, MD,1-3 \\ Emmanuel Moyse, MD, ${ }^{1,2}$ and Carlo Giussani, MD, PhD ${ }^{4}$
}

${ }^{1}$ Pôle Neuroscience (Neurochirurgie), Centre Hospitalo-Universitaire de Toulouse, ${ }^{2}$ Université de Toulouse, UPS; ${ }^{3}$ Centre de Recherche Cerveau et Cognition (CNRS; CerCo), Toulouse, France; and ${ }^{4}$ Neurosurgery, Dipartimento di Medicina e Chirurgia, Università degli Studi di Milano-Bicocca, Ospedale San, Gerardo, Monza, Italy

\begin{abstract}
OBJECTIVE Electrostimulation in awake brain mapping is widely used to guide tumor removal, but methodologies can differ substantially across institutions. The authors studied electrostimulation brain mapping data to characterize the variability of the current intensity threshold across patients and the effect of its variations on the number, type, and surface area of the essential language areas detected.
\end{abstract}

METHODS Over 7 years, the authors prospectively studied 100 adult patients who were undergoing intraoperative brain mapping during resection of left hemisphere tumors. In all 100 cases, the same protocol of electrostimulation brain mapping (a controlled naming task-bipolar stimulation with biphasic square wave pulses of 1-msec duration and $60-\mathrm{Hz}$ trains, maximum train duration $6 \mathrm{sec}$ ) and electrocorticography was used to detect essential language areas.

RESULTS The minimum positive thresholds of stimulation varied from patient to patient; the mean minimum intensity required to detect interference was $4.46 \mathrm{~mA}$ (range 1.5-9 mA), and in a substantial proportion of sites (13.5\%) interference was detected only at intensities above $6 \mathrm{~mA}$. The threshold varied within a given patient for different naming areas in $22 \%$ of cases. Stimulation of the same naming area with greater intensities led to slight changes in the type of response in $19 \%$ of cases and different types of responses in $4.5 \%$. Naming sites detected were located in subcentimeter cortical areas $\left(50 \%\right.$ were less than $\left.20 \mathrm{~mm}^{2}\right)$, but their extent varied with the intensity of stimulation. During a brain mapping session, the same intensity of stimulation reproduced the same type of interference in $94 \%$ of the cases. There was no statistically significant difference between the mean stimulation intensities required to produce interfereince in the left inferior frontal lobe (Broca's area), the supramarginal gyri, and the posterior temporal region.

CONCLUSIONS Intrasubject and intersubject variations of the minimum thresholds of positive naming areas and changes in the type of response and in the size of these areas according to the intensity used may limit the interpretation of data from electrostimulation in awake brain mapping. To optimize the identification of language areas during electrostimulation brain mapping, it is important to use different intensities of stimulation at the maximum possible currents, avoiding afterdischarges. This could refine the clinical results and scientific data derived from these mapping sessions. https://thejns.org/doi/abs/10.3171/2016.4.JNS152434

KEY WORDS electrostimulation; language; brain tumor; brain mapping; awake surgery; diagnostic and operative techniques; oncology

$\mathrm{E}$ LECTROSTIMULATION in awake patients has been used intensively in neurosurgery to map language functions, and a large body of work has been published on the subject. ${ }^{13,24,32}$ Debates have primarily focused on the types of tasks considered the best predictors of language outcome, and naming has long been used for this purpose..$^{18}$ Although the technique has become popular over the last 20 years, it must be acknowledged that the practice of electrostimulation for brain mapping relies mainly on empirical data. Studies that have investigated the optimal stimulation parameters for human brain mapping (intensity of stimulation, duration of stimulation, extent of the stimulated area) are relatively rare. . $^{8,15,26,28,36,38}$ However, the parameter of intensity of stimulation alone may vary across individuals ${ }^{26}$ or with age ${ }^{7}$ or with the presence of cerebral edema ${ }^{38}$ for instance, and questions about this most basic mapping parameter are still numerous. What is the "optimal" intensity to detect essential language sites for naming? Does this optimal intensity of stimulation vary with the task used or across the cortex (some language areas being detected at different levels of intensity)? Does the stimulation threshold differ across the exposed cortex (frontal 
versus temporal, for instance)? Could the variation of the level of intensity at a given essential site lead to a different type of response (e.g., anomia at $3 \mathrm{~mA}$ but speech arrest at $5 \mathrm{~mA}$ )? These questions are important if a standardized stimulation protocol is to be used by all practitioners to improve the accuracy of the procedure, the safety of the patient, and the comparability of the resulting scientific data among the different neurosurgical centers worldwide.

In 2008, we decided to design a prospective study that would include a substantial number of patients studied with the same protocol over many years. This protocol, based at that time on our 10 years' experience in awake brain mapping by electrostimulation, ${ }^{31}$ needed be simple enough to be applicable to a large number of patients and reproducible by other neurosurgical teams. Although Penfield and Roberts ${ }^{23,24}$ used at least 3 different stimulators, the works of Ojemann et al. ${ }^{18}$ popularized the use of bipolar electrodes with biphasic square wave pulses, each of 1-msec duration, at $60 \mathrm{~Hz}$ with a maximum stimulation duration of 4 seconds. These standard parameters of stimulation were used in this study, which was intended to clarify 3 main points: 1) What is the minimum intensity required to observe naming interference in awake patients? 2) Does the type of naming interference observed differ according to the level of stimulation? 3) Can we accurately discern the real surface area of these interferences? In addition to these main points, we also wanted to test secondary issues, such as the range of intensities evoking interference according to the gyri simulated and whether the type of interference was reproducible over a given area during a brain mapping session at constant intensity.

\section{Methods}

\section{Inclusion Criteria}

This was a prospective study. From the outset, our plan was to close the study once 100 brain mappings had been included. We studied electrostimulation data from brain mappings performed for the removal of recently discovered brain lesions in conscious individuals (mean age 52 years, range 20-79 years, SD 6 years, 42 women) (Table 1). The lesions included 29 WHO Grade I and II gliomas, 46 WHO Grade III and IV gliomas, 8 arteriovenous malformations or cavernomas, 16 metastases, and 1 atypical meningioma, and the mean time between the first clinical sign and the operation was 35 days (range 14-86 days). Data from the brain mapping sessions were prospectively collected by the same team using the same protocol throughout the 7 years of the study (June 2008-April 2015). The National Consultative Ethics Committee (Comité Consultatif National d'Ethique) of INSERM (Institut National de la Santé et de la Recherche Médicale) gave its approval for the storage of patients' data and procedures to ensure preservation of their anonymity. All of the patients and in most cases also their families gave their informed consent for study of their functional areas by direct brain mapping. All patients underwent an assessment of handedness ${ }^{22}$ and standardized, preoperative language tests (administered by our speech therapist) as follows: visual naming using the DO80 test (this test was also performed postoperatively in all cases, between 2 days to 2 weeks after the operation); ${ }^{5}$ tests of written, auditory, and visual comprehension abilities; and oral fluency, reading, dictation, repetition, written transcription, and object handling tests. ${ }^{16}$

\section{Exclusion Criteria}

Not all consecutive patients who underwent awake surgery were included in this study. Ten dysphasic patients (with an error rate greater than 10\% in the DO80 naming test) and patients with chronic epilepsy were initially excluded. Eleven additional patients were excluded subsequently because they were unable to perform the naming task accurately for various reasons: 2 patients were unable to awake correctly for brain mapping; 6 patients experienced spontaneous naming difficulties between the preoperative language test and the operation; and in 2 patients brain mapping was constrained by clinical requirements (brain swelling in 1 case and subarachnoid hemorrhage at the dura opening in the other). One patient younger than 18 years old was also excluded. Age can be a confounding factor for calculating the intensity of stimulation during brain mapping sessions. ${ }^{19}$

\section{Anesthetic Protocol for Awake Craniotomy}

Patients were all operated on using the "awake surgery" technique. Anesthetic drugs can, in theory, interfere with stimulation thresholds. Our objective during brain mapping was to avoid any anesthetic drugs. One hour before admission to the operating room, a patch containing a eutectic mixture of prilocaine $(2.5 \mathrm{mg} / \mathrm{g})$ and lidocaine $(2.5 \mathrm{mg} / \mathrm{g})$ (EMLA) was applied in the supraorbital and auriculotemporal regions. Lidocaine $1 \%$ with epinephrine 1:100,000 was infiltrated to block the supraorbital, auriculotemporal, and occipital nerves. Additionally, the Mayfield head holder (Ohio Medical) pin site and the surgical skin incision line were infiltrated. Sedation with spontaneous respiration was provided by continuous infusion of propofol (1-3 mg/kg/hr). Fentanyl (1-3 $\mu \mathrm{g} / \mathrm{kg} / \mathrm{hr})$ or remifentanil $(0.01-0.25 \mu \mathrm{g} / \mathrm{kg} / \mathrm{hr})$ was used for analgesia. The depth of procedural sedation was adjusted to maintain acceptable comfort and stable vital signs in the patient. Propofol infusion was stopped during the dural opening (around 10 minutes before brain mapping). Once the cortical mapping procedure was completed, patients were put back to sleep using the same protocol for the rest of the operation.

\section{Cortical Mapping}

\section{Basic Procedure}

Our standard protocol has been described elsewhere. ${ }^{29,31}$ Briefly, a neuronavigational system was used in all 100 cases to guide tumor removal, and intraoperative cortical stimulation was used to localize areas of functional cortex. Anatomical structures (gyri and sulci) were identified according to the neuronavigational data and the visual identification of the shape of gyri and sulci (i.e., 3D anatomy of the circumvolutions). Before starting a direct cortical stimulation procedure, we chose a substantial number of sites on the brain surface. Each site was separated from the others by $3 \mathrm{~mm}$. The number of stimulation sites varied among patients depending on the size and the location of the craniotomy. 
TABLE 1. Summary of patient data

\begin{tabular}{|c|c|c|c|c|c|c|c|}
\hline \multirow{2}{*}{$\begin{array}{l}\text { Case } \\
\text { No. }\end{array}$} & \multirow[b]{2}{*}{ Sex } & \multirow{2}{*}{$\begin{array}{l}\text { Age } \\
\text { (yrs) }\end{array}$} & \multirow{2}{*}{$\begin{array}{c}\text { Main } \\
\text { Symptoms }\end{array}$} & \multirow{2}{*}{$\begin{array}{l}\text { Tumor } \\
\text { Location }\end{array}$} & \multirow[b]{2}{*}{ Pathology } & \multicolumn{2}{|c|}{ D080 Score* } \\
\hline & & & & & & Preop & Postop \\
\hline 1 & $\mathrm{~F}$ & 60 & Seizures & $A G, S M G$ & Metastasis & 75 & 76 \\
\hline 2 & M & 64 & Seizures & MTG & Astrocytoma, Gr III & 78 & 74 \\
\hline 3 & $\mathrm{~F}$ & 35 & Headaches & SFG & Astrocytoma, Gr II & 78 & 79 \\
\hline 4 & M & 67 & Seizures & MTG, ITG & Astrocytoma, Gr III & 76 & 77 \\
\hline 5 & $\mathrm{~F}$ & 66 & Headaches & Insula & Glioblastoma & 78 & 73 \\
\hline 6 & $M$ & 71 & Gerstmann syndrome & SMG & Metastasis & 76 & 74 \\
\hline 7 & $\mathrm{~F}$ & 45 & Seizures & SFG, MFG & Astrocytoma, Gr III & 77 & 78 \\
\hline 8 & $M$ & 60 & Seizures & STG, MTG & Glioblastoma & 77 & 79 \\
\hline 9 & M & 57 & Motor defect & Insula & Ependymoma, Gr II & 70 & 75 \\
\hline 10 & $\mathrm{~F}$ & 31 & Seizures & MFG, IFG & Cavernoma & 80 & 80 \\
\hline 11 & $M$ & 36 & Headaches & RF, SMG & Cavernoma & 78 & 79 \\
\hline 12 & M & 54 & Asymptomatic & IFG & Astrocytoma, Gr II & 76 & 73 \\
\hline 13 & M & 28 & Seizures & ITG & Ependymoma, Gr III & 79 & 77 \\
\hline 14 & M & 67 & Seizures & MTG & Glioblastoma & 78 & 72 \\
\hline 15 & $\mathrm{~F}$ & 25 & Seizures & STG & Ganglioglioma, Gr I & 79 & 79 \\
\hline 16 & M & 50 & Seizures & IFG & Glioblastoma & 73 & 69 \\
\hline 17 & $\mathrm{~F}$ & 31 & Headaches & MFG, IFG & Astrocytoma, Gr II & 79 & 76 \\
\hline 18 & M & 59 & Seizures & STG & Astrocytoma, Gr III & 75 & 71 \\
\hline 19 & M & 54 & Seizures & STG, MTG & Astrocytoma, Gr III & 80 & 77 \\
\hline 20 & $\mathrm{~F}$ & 54 & Speech disorders & RF, SMG & Metastasis & 78 & 73 \\
\hline 21 & M & 49 & Asymptomatic & IFG & Oligodendroglioma, Gr II & 80 & 80 \\
\hline 22 & M & 50 & Seizures & RF, IFG & Glioblastoma & 72 & 65 \\
\hline 23 & $\mathrm{~F}$ & 69 & Seizures & SMG & Glioblastoma & 77 & 75 \\
\hline 24 & $\mathrm{~F}$ & 38 & Seizures & MTG & Astrocytoma, Gr II & 77 & 78 \\
\hline 25 & $M$ & 79 & Seizures & SMG & Glioblastoma & 77 & 71 \\
\hline 26 & M & 41 & Seizures & STG, SMG & Astrocytoma, Gr II & 78 & 73 \\
\hline 27 & M & 68 & Speech disorders & AG, SMG & Glioblastoma & 76 & 77 \\
\hline 28 & M & 47 & Seizures & SMG & Ganglioglioma, Gr I & 79 & 74 \\
\hline 29 & M & 70 & Seizures & STG & Glioblastoma & 79 & 73 \\
\hline 30 & $M$ & 53 & Seizures & SMG & Metastasis & 78 & 79 \\
\hline 31 & M & 76 & Seizures & MTG & Astrocytoma, Gr II & 78 & 75 \\
\hline 32 & M & 57 & Asymptomatic & STG, MTG & Astrocytoma, Gr II & 75 & 74 \\
\hline 33 & $\mathrm{~F}$ & 47 & Seizures & IFG, RF & Oligodendroglioma, Gr III & 77 & 80 \\
\hline 34 & M & 54 & Seizures & $A G$ & Glioblastoma & 78 & 76 \\
\hline 35 & $M$ & 74 & Speech disorders & STG, SMG & Metastasis & 80 & 80 \\
\hline 36 & M & 25 & Sensory defect & MFG & Cavernoma & 80 & 80 \\
\hline 37 & $\mathrm{~F}$ & 57 & Seizures & IFG & Glioblastoma & 78 & 79 \\
\hline 38 & M & 36 & Seizures & SMG & Astrocytoma, Gr III & 80 & 80 \\
\hline 39 & M & 54 & Seizures & MFG & Glioblastoma & 80 & 80 \\
\hline 40 & M & 44 & Seizures & SFG & Astrocytoma, Gr III & 79 & 80 \\
\hline 41 & $\mathrm{~F}$ & 69 & Headaches & MTG & Glioblastoma & 77 & 75 \\
\hline 42 & M & 47 & Seizures & RF, SFG & Metastasis & 80 & 80 \\
\hline 43 & M & 66 & Sensory defect & RF & Glioblastoma & 72 & 73 \\
\hline 44 & $\mathrm{~F}$ & 64 & Asymptomatic & RF & Metastasis & 80 & 80 \\
\hline 45 & $\mathrm{~F}$ & 60 & Facial palsy & RF, IFG & Glioblastoma & 77 & 76 \\
\hline 46 & $M$ & 28 & Memory troubles & STG, hippocampus & Ganglioglioma, Gr I & 79 & 80 \\
\hline 47 & M & 49 & Seizures & STG, MTG & Astrocytoma, Gr III & 80 & 78 \\
\hline 48 & $\mathrm{~F}$ & 32 & Seizures & MTG & Cavernoma & 77 & 75 \\
\hline
\end{tabular}


» CONTINUED FROM PAGE 1643

TABLE 1. Summary of patient data

\begin{tabular}{|c|c|c|c|c|c|c|c|}
\hline \multirow{2}{*}{$\begin{array}{l}\text { Case } \\
\text { No. }\end{array}$} & \multirow[b]{2}{*}{ Sex } & \multirow{2}{*}{$\begin{array}{l}\text { Age } \\
\text { (yrs) }\end{array}$} & \multirow{2}{*}{$\begin{array}{c}\text { Main } \\
\text { Symptoms }\end{array}$} & \multirow{2}{*}{$\begin{array}{l}\text { Tumor } \\
\text { Location }\end{array}$} & \multirow[b]{2}{*}{ Pathology } & \multicolumn{2}{|c|}{ D080 Score* } \\
\hline & & & & & & Preop & Postop \\
\hline 49 & $M$ & 21 & Seizures & IFG & Xanthoastrocytoma, Gr I & 80 & 80 \\
\hline 50 & $\mathrm{~F}$ & 35 & Headaches & SFG & Astrocytoma, Gr III & 78 & 79 \\
\hline 51 & $\mathrm{~F}$ & 77 & Speech disorders & MFG, IFG & Glioblastoma & 74 & 70 \\
\hline 52 & $\mathrm{~F}$ & 40 & Seizures & IFG & Atypical meningioma & 80 & 80 \\
\hline 53 & $\mathrm{~F}$ & 58 & Headaches & STG, MTG & Glioblastoma & 78 & 80 \\
\hline 54 & $\mathrm{~F}$ & 79 & Seizures & MFG, IFG & Glioblastoma & 77 & 74 \\
\hline 55 & $M$ & 40 & Headaches & SFG & Metastasis & 80 & 80 \\
\hline 56 & M & 57 & Seizures & MFG & Oligodendroglioma, Gr II & 78 & 77 \\
\hline 57 & M & 43 & Hemorrhage & STG, hippocampus & Astrocytoma, Gr II & 76 & 74 \\
\hline 58 & $\mathrm{~F}$ & 67 & Speech disorders & SMG & Oligodendroglioma, Gr III & 78 & 74 \\
\hline 59 & $\mathrm{~F}$ & 52 & Hemiparesis & SMG & Ependymoma, Gr II & 80 & 79 \\
\hline 60 & M & 50 & Speech disorders & MFG & Glioblastoma & 75 & 76 \\
\hline 61 & M & 58 & Apraxia & SMG & Glioblastoma & 79 & 73 \\
\hline 62 & $\mathrm{~F}$ & 33 & Seizures & ITG & Cavernoma & 80 & 80 \\
\hline 63 & M & 36 & Seizures & MFG & Oligodendroglioma, Gr II & 78 & 80 \\
\hline 64 & $\mathrm{~F}$ & 66 & Speech disorders & IFG & Glioblastoma & 79 & 74 \\
\hline 65 & M & 72 & Seizures & $A G$ & Metastasis & 79 & 80 \\
\hline 66 & $\mathrm{~F}$ & 64 & Seizures & MTG & Glioblastoma & 72 & 71 \\
\hline 67 & $\mathrm{~F}$ & 64 & Seizures & $A G$ & Metastasis & 78 & 75 \\
\hline 68 & $\mathrm{~F}$ & 55 & Motor defect & SFG, MFG & Metastasis & 78 & 78 \\
\hline 69 & $\mathrm{~F}$ & 67 & Seizures & STG, MTG & Glioblastoma & 77 & 76 \\
\hline 70 & M & 63 & Motor defect & IFG & Glioblastoma & 79 & 77 \\
\hline 71 & M & 20 & Seizures & STG & Astrocytoma, Gr I & 76 & 76 \\
\hline 72 & M & 68 & Seizures & IFG & Oligodendroglioma, Gr II & 80 & 78 \\
\hline 73 & M & 31 & Asymptomatic & STG, MTG & Oligodendroglioma, Gr II & 80 & 80 \\
\hline 74 & $M$ & 57 & Speech disorders & STG, SMG & Astrocytoma, Gr II & 78 & 75 \\
\hline 75 & M & 73 & Seizures & STG, MTG & Cavernoma & 76 & 75 \\
\hline 76 & M & 22 & Seizures & STG, MTG & Astrocytoma,Gr II & 79 & 78 \\
\hline 77 & $\mathrm{~F}$ & 52 & Speech disorders & STG, SMG & Cavernoma & 79 & 80 \\
\hline 78 & M & 46 & Headaches & SFG & Cavernoma & 80 & 80 \\
\hline 79 & $\mathrm{~F}$ & 37 & Seizures & MTG & Ganglioglioma, Gr I & 80 & 80 \\
\hline 80 & $M$ & 22 & Seizures & MFG & Metastasis & 80 & 80 \\
\hline 81 & $\mathrm{M}$ & 56 & Hemiparesis & SMG & Metastasis & 78 & 79 \\
\hline 82 & $\mathrm{~F}$ & 42 & Hemiparesis & $\mathrm{RF}$ & Metastasis & 80 & 80 \\
\hline 83 & $\mathrm{~F}$ & 57 & Hemiparesis & SFG & Glioblastoma & 77 & 78 \\
\hline 84 & M & 67 & Asymptomatic & RF, IFG & Glioblastoma & 75 & 75 \\
\hline 85 & $M$ & 62 & Speech disorders & SMG & Glioblastoma & 72 & 65 \\
\hline 86 & $\mathrm{~F}$ & 46 & Seizures & STG, MTG, insula & Astrocytoma, Gr II & 78 & 76 \\
\hline 87 & $\mathrm{~F}$ & 68 & Seizures & IFG & Astrocytoma, Gr III & 76 & 73 \\
\hline 88 & $\mathrm{~F}$ & 37 & Speech disorders & Insula & Astrocytoma, Gr III & 77 & 80 \\
\hline 89 & $\mathrm{~F}$ & 47 & Seizures & MFG, IFG & Glioblastoma & 72 & 79 \\
\hline 90 & $M$ & 68 & Speech disorders & $A G, S M G$ & Glioblastoma & 74 & 74 \\
\hline 91 & M & 48 & Seizures & IFG, MFG & Oligodendroglioma, Gr II & 77 & 76 \\
\hline 92 & $\mathrm{~F}$ & 30 & Seizures & SFG & Ganglioglioma, Gr I & 79 & 79 \\
\hline 93 & $\mathrm{~F}$ & 68 & Seizures & IFG, MFG & Glioblastoma & 72 & 69 \\
\hline 94 & M & 51 & Seizures & SFG, MFG & Astrocytoma, Gr II & 80 & 80 \\
\hline 95 & $M$ & 35 & Seizures & $\mathrm{RF}$ & Oligodendroglioma, Gr II & 80 & 80 \\
\hline
\end{tabular}


» CONTINUED FROM PAGE 1644

TABLE 1. Summary of patient data

\begin{tabular}{rrrlllcc}
\hline $\begin{array}{c}\text { Case } \\
\text { No. }\end{array}$ & Sex & $\begin{array}{c}\text { Age } \\
(\mathrm{yrs})\end{array}$ & \multicolumn{1}{c}{$\begin{array}{c}\text { Main } \\
\text { Symptoms }\end{array}$} & \multicolumn{1}{c}{$\begin{array}{c}\text { Tumor } \\
\text { Location }\end{array}$} & Pathology & \multicolumn{2}{c}{ DO80 Score $^{*}$} \\
\hline 96 & M & 47 & Seizures & RF, MFG & Metastasis & Preop & Postop \\
\hline 97 & M & 42 & Seizures & IFG, MFG, SFG & Astrocytoma, Gr II & 80 & 80 \\
\hline 98 & F & 69 & Seizures & AG & Metastasis & 72 & 61 \\
\hline 99 & F & 69 & Speech disorders & MTG & Glioblastoma & 73 & 72 \\
\hline 100 & F & 77 & Speech disorders & MFG, IFG & Glioblastoma & 72 & 69 \\
\hline
\end{tabular}

$\mathrm{AG}=$ angular gyrus; $\mathrm{Gr}$ = Grade; IFG = inferior frontal gyrus; $\mathrm{MFG}$ = middle frontal gyrus; $\mathrm{MTG}$ = middle temporal gyrus; $\mathrm{RF}$ = rolandic fissure; SFG = superior frontal gyrus; $S M G$ = supramarginal gyrus; $S T G$ = superior temporal gyrus

* Visual naming test D080. ${ }^{5}$

\section{Naming Task}

We used a standard naming task. Images were chosen for their high familiarity, with a mean familiarity value above 2 (Table 2) according to the image familiarity ratings of Snodgrass and Vanderwart. ${ }^{33}$ During the procedure, images were randomly selected from this set, and stimulation was applied just before the image was displayed and during the picture presentation. We refined this brain mapping image set over more than 10 years, presenting only highly familiar stimuli to patients. ${ }^{30}$

\section{Protocol Used in This Study}

Two types of thresholds were defined: the minimum positive threshold necessary to produce interference in a cortical area (the main threshold used in this study) and the "afterdischarge threshold," a current intensity producing afterdischarges, sources of mislocalizations during brain mapping.

A checklist was established for each brain mapping session, based on 3 main parameters: the minimum positive threshold level of each positive site; its surface area; and its changes in terms of surface area and type of interference detected when the area was stimulated above its threshold level.

The cortex was directly stimulated using the bipolar electrode separated by $3 \mathrm{~mm}$ from the Nimbus cortical stimulator (1-mm electrodes, Newmedic) with biphasic square wave pulses of $1-\mathrm{msec}$ duration and $60-\mathrm{Hz}$ trains. The maximum train duration of each stimulation was 6 seconds in total (around 1 or 2 seconds before picture presentation and 4 or 5 seconds after). The "afterdischarge threshold" was determined by electrocorticography using a strip electrode. Although this point remains controversial, ${ }^{11,26}$ the "afterdischarge threshold" has been found to change little from site to site in a single short mapping session. ${ }^{17}$ The level of electrostimulation was always kept $1 \mathrm{~mA}$ below the level expected to cause electrical diffusion and afterdischarges so as to ensure that the stimulated area remained accurately localized on the area of cortex under study. If any afterdischarge (or epileptic seizure) was detected, the protocol was adapted to the "afterdischarge threshold" found.

During the mapping session, cortical stimulation was performed over the whole exposed brain surface at $5 \mathrm{~mA}$, at $7 \mathrm{~mA}$ (excluding the positive sites already found at 5
TABLE 2. Mean and standard deviation of the familiarity rating of the images used for the naming test ${ }^{*}$

\begin{tabular}{|c|c|c|c|}
\hline \multirow{2}{*}{$\begin{array}{c}\text { Image } \\
\text { No. }\end{array}$} & \multirow[b]{2}{*}{ Description } & \multicolumn{2}{|c|}{ Familiarity } \\
\hline & & Mean & SD \\
\hline 1 & Airplane & 3.78 & 0.99 \\
\hline 2 & Arrow & 3.38 & 1.23 \\
\hline 3 & Apple & 3.98 & 1.08 \\
\hline 4 & Ball & 3.20 & 1.21 \\
\hline 5 & Banana & 3.65 & 1.04 \\
\hline 6 & Bed & 4.72 & 0.77 \\
\hline 7 & Belt & 4.12 & 1.05 \\
\hline 8 & Bicycle & 3.78 & 1.04 \\
\hline 9 & Bird & 3.63 & 1.16 \\
\hline 10 & Bus & 4.50 & 0.74 \\
\hline 11 & Butterfly & 2.92 & 1.17 \\
\hline 12 & Cake & 4.02 & 1.06 \\
\hline 13 & Carrot & 3.55 & 0.97 \\
\hline 14 & Chair & 4.58 & 0.86 \\
\hline 15 & Cherry & 3.38 & 1.18 \\
\hline 16 & Car & 4.70 & 0.60 \\
\hline 17 & Corn & 3.50 & 1.05 \\
\hline 18 & Couch & 4.40 & 0.74 \\
\hline 19 & Duck & 2.75 & 1.11 \\
\hline 20 & Frog & 2.48 & 1.05 \\
\hline 21 & Guitar & 3.58 & 1.09 \\
\hline 22 & Helicopter & 2.55 & 1.12 \\
\hline 23 & Lemon & 3.25 & 1.22 \\
\hline 24 & Mouse & 2.45 & 1.02 \\
\hline 25 & Onion & 3.32 & 1.31 \\
\hline 26 & Pipe & 2.90 & 1.14 \\
\hline 27 & Table & 4.35 & 0.88 \\
\hline 28 & Scissors & 3.98 & 0.99 \\
\hline 29 & Tree & 4.68 & 0.61 \\
\hline 30 & Watch & 4.58 & 0.73 \\
\hline
\end{tabular}

* Images were chosen for their high familiarity (mean familiarity $>2$ ) according to the familiarity ratings of Snodgrass and Vanderwart as published in their 1980 article..$^{33}$ The mean and SD values shown were extracted from Appendix $\mathrm{B}$ of that paper and are based on a 5-point rating scale, with 5 indicating "very familiar" and 1 , "very unfamiliar." 
$\mathrm{mA}$ ), and finally at $10 \mathrm{~mA}$ (excluding the positive sites already found at 5 and $7 \mathrm{~mA}$ ). This step defined 3 approximate stimulation levels for the positive sites.

Once this step was completed, all the positive areas found were tested again (at levels below their positive threshold stimulation levels) with the aim of refining the exact level of current necessary to detect the positive cortical area found and determining the minimum positive threshold. For instance, if a positive area was found at 7 $\mathrm{mA}$, the area was tested at $6.5 \mathrm{~mA}, 6 \mathrm{~mA}, 5.5 \mathrm{~mA}$, and so on for all the positive area found. If a positive area was detected at $5 \mathrm{~mA}$, it was tested at $4.5 \mathrm{~mA}, 4 \mathrm{~mA}, 3.5 \mathrm{~mA}$, and so on until its minimum positive threshold was found.

Although this can be debated, we thought that the direct advantage of this protocol $(5,7$, and $10 \mathrm{~mA})$ was to shorten the time necessary for a brain mapping. The postulate underlying this approach is that an area identified at its minimum intensity threshold would also be positive at higher thresholds. Stimulating at 7 and $10 \mathrm{~mA}$ directly allows all the standard increments $(0.5 \mathrm{~mA}$ by $0.5 \mathrm{~mA})$ between 5 and $7 \mathrm{~mA}$, and then $10 \mathrm{~mA}$, to be skipped. We also considered that patient fatigue during language mapping can be an important factor and that all modifications that could reduce the time of brain mapping would be welcome.

It is worth remembering that the number of naming interferences found was usually low (1-3 in many brain mapping sessions). Thus, this protocol was usually completed in less than 15-20 minutes.

\section{Prospective Collection of Data}

Video recordings were made of all brain mapping sessions. Intraoperative photographs of the brain were taken, showing the sites validated according to this procedure. In the hours after the operation, all data regarding brain mapping results were stored in an Excel database and each positive cortical point found was labeled with Talairach coordinates. ${ }^{30}$

\section{Criteria for Positive Interference}

Only unequivocal brain mapping results were included in this study. The patient's performance in the absence of stimulation was regularly measured throughout the testing, as further assurance that changes during stimulation were not random events. Nonreproducible interferences were not included in this study. The reproducibility criterion was 3/3 (i.e., 3 interferences to validate a cortical site as "positive" for the naming task). When a site had a reproducibility of $2 / 3$ we stimulated it at least 1 more time: reproducibility criteria of $3 / 4$ (or 4/5) were validated but not $2 / 4$ or $3 / 5$. At least 3 trials were performed on positive sites.

No initial (i.e., preoperative) instruction was given to the patients to report unintelligibility, but the patients were systematically debriefed during the operation when they made naming errors. The language interferences found in the pre- and postcentral gyrus (considered as language interferences due to blockade of articulatory mechanisms) were not included in the final analysis, because they can be considered nonspecific.

\section{Classification of Naming Interferences}

We classified the naming interferences found in 5 cate- gories as: 1) global behavior arrest (patient stopped talking during stimulation); 2) anomia (patient said "yes, I know... yes...I know but I can't find the word..." and, as soon as stimulation was stopped, the target word was produced); 3) phonological interference (the target word seemed to be identified but was not correctly pronounced; for instance during stimulation one patient said "c'est un rococopder" ["it's a rococopder"] when the target was "helicopter" [hélicoptère]); 4) semantic interference (the target word was not identified, and patients showed verbal paraphasia suggesting semantic errors-for instance, 1 patient said "this is a banana" when a car was presented); or 5) various other interferences, classified as "hesitation" (i.e., upon stimulation, patients did not produce the correct response at once, hesitated, and were not sure of their final response). This classification system can be debated. Although other authors have used slightly different classifications, the basis of many classifications consists of anomia, speech arrest, and semantic and phonologic errors. ${ }^{4}$ The main topic under study here was not directly based on the type of interference found, and we considered that this classification suited the main objectives of the study.

\section{Evaluation of the Interference Category According to the Intensity}

The identified positive areas were stimulated above their minimum positive threshold level to test whether the type of response obtained at a given intensity remained at higher intensity. To avoid excessive testing time and patient fatigue, all positive areas found under $7 \mathrm{~mA}$ were tested at 7 and $10 \mathrm{~mA}$ (e.g., when a positive area was detected at $4.5 \mathrm{~mA}$, it was tested again at 7 and $10 \mathrm{~mA}$ to observe any changes in the type of response obtained). Others were tested at $10 \mathrm{~mA}$ (e.g., when an area was detected at 7.5 $\mathrm{mA}$, it was tested again at $10 \mathrm{~mA}$ ).

\section{Evaluation of the Area of the Interference Site}

When a functional site was found, it was marked by a sterile tag before moving on to the next test site. Because the distance between the tips of the bipolar electrode was $3 \mathrm{~mm}$, we considered this distance $(3 \mathrm{~mm})$ the minimum spatial resolution of our electrosimulation technique. Once the minimum positive threshold of an area was found, we tested the cortical surface of the area detected. The cortical areas located in the immediate vicinity were tested using the same intensity to find out whether they were positive or negative. Once a surface had been defined, the 2 sides of the area (considered as a rectangle) were measured and its surface area was calculated. The minimum surface area detected by our mapping was a square of $3 \times 3 \mathrm{~mm}$ (i.e., $9 \mathrm{~mm}^{2}$ ) but larger surfaces were also detected.

\section{Extension of the Area of the Interference Site When the Intensity Increased}

The immediate areas located close to the interference area were also tested using $7 \mathrm{~mA}$ and/or $10 \mathrm{~mA}$ when the positive threshold level was below these intensities. We tested whether the cortical surface area was extended with higher intensities (for instance, when an interference area was positive at $5.5 \mathrm{~mA}$, the cortex located immediately close to it was stimulated at $7 \mathrm{~mA}$ and $10 \mathrm{~mA}$ to test whether the positive area increased with increasing current intensity). 


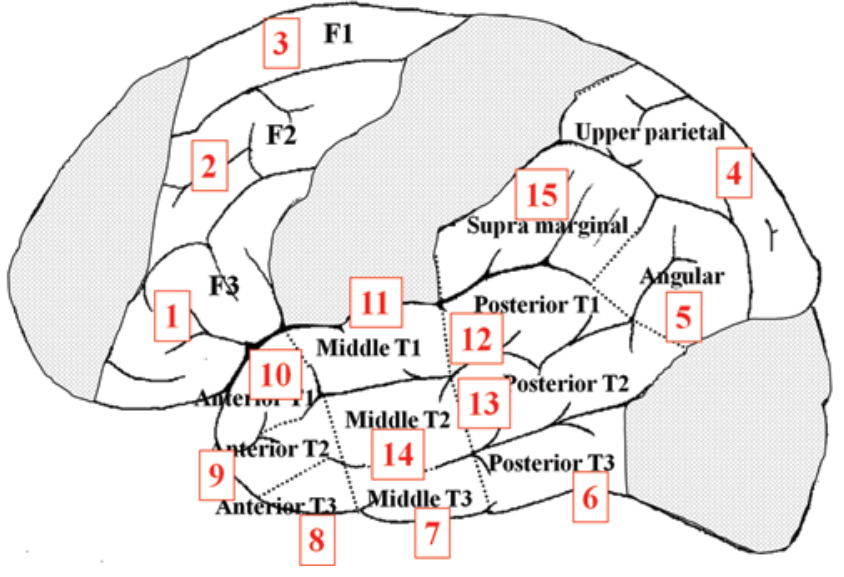

FIG. 1. Scheme of the gyral anatomy used to perform the statistical analysis. Several regions were defined, from 1 to 15. As the terms "Broca's" or "Wernicke's" regions were imprecise and not very informative, we decided to perform statistical analysis to define regions by using the gyral and sulcal anatomy. For instance, the supramarginal gyrus was considered as a region, as was the angular gyrus. Large gyri, such as the temporal gyri, for example, were arbitrarily divided into 3 segments by drawing an imaginary line extending the pre- and postcentral sulci. All negative cortical sites were also included in this system of cortical localization. Figure is available in color online only.

\section{Statistical Analysis}

Descriptive statistics were used to compute the total number of positive sites per patient, the intensity of stimulation evoking interference in each positive site, and the minimum intensity inducing interference in all positive sites for each patient.

The mean minimum intensity of stimulation was compared across cortical regions by first grouping the positive sites according to our scheme for dividing up gyri (see Fig. 1).

The influence of the locations of positive sites on the stimulation intensities was assessed by means of a 1-way ANOVA and post hoc pairwise comparisons through Student t-tests. A similar approach was used to study the influence of the stimulation intensity on the type of interference induced by the stimulation.

\section{Results}

Overall, the data set comprised 3790 stimulated cortical sites obtained from cortical mapping in 100 patients (average per patient 38 sites, range 8-90 sites). Afterdischarges were seen in 9 cortical mapping sessions-for 4 $\mathrm{mA}$ ( 1 case), $8 \mathrm{~mA}$ ( 5 cases), $8.5 \mathrm{~mA}$ (1 case), $9.5 \mathrm{~mA}$ (1 case), and $10 \mathrm{~mA}$ (1 case). The other 91 brain mappings were all conducted up to $10 \mathrm{~mA}$. Intraoperative epileptic seizures were observed in 4 cases ( 1 generalized, 3 partial) and were rapidly controlled by irrigating the cortex with cold Ringer's lactate. Of these 4 cases, 3 were detected with afterdischarges; the last patient had a partial seizure during stimulation of the superior frontal gyrus at $10 \mathrm{~mA}$. We waited for a few minutes and repeated this brain mapping at a lower intensity. No stimulation was performed above the "afterdischarge threshold" in these 9 patients.

In 20 patients, no language interference sites were de-

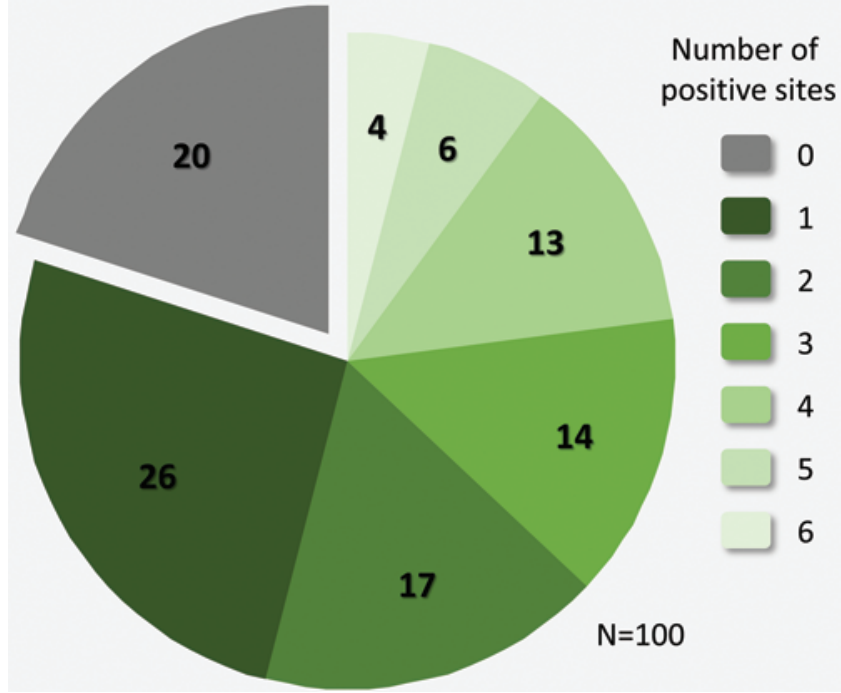

FIG. 2. Pie chart showing the number of patients for whom $0,1,2,3,4$, 5 , or 6 naming sites were identified. No language site was evidenced in 20 patients, despite the fact that stimulations were performed in presumed language regions of the left hemisphere. In line with most electrostimulation brain mapping studies, most patients (90 of 100) had less than 5 sites detected. Figure is available in color online only.

tected, even with the maximum intensity of stimulation of $10 \mathrm{~mA}$. In the other 80 patients, at least 1 site was detected by electrostimulation (Fig. 2). Overall, 208 naming interference sites (5.5\% of the total number of sites stimulated) were detected. According to our classification, we found (with the minimum positive threshold) 55 speech arrest, 61 anomia, 62 phonemic, 20 semantic, and 10 "hesitation" interferences.

In 22 mapping sessions (22\% of the cases), various current intensities were needed at different cortical sites to obtain language interferences. To detect all language interferences, 2 different intensities were necessary in 12 mapping sessions, 3 different intensities in 5, and 4 different intensities in 2.

Although it is beyond the scope of this paper to discuss the aspects of the postoperative language outcomes in detail according to brain mapping results, we noticed that in 16 patients the number of images named in the DO80 visual naming test decreased by 5\%-10\% compared with preoperative data. In 1 patient, the number of images named postoperatively decreased by $15 \%$ compared with preoperative DO80 results. In 2 patients, the number of images named increased after the operation by more than $5 \%$. In other patients, the number of images named during the visual naming test either improved after the operation or remained stable at around $\pm 5 \%$. Because many of the patients had postoperative radiotherapy and chemotherapy, the time course of the language outcome was not systematically studied afterward.

\section{Minimum Positive Thresholds for Naming Interference}

The mean intensity required to detect the 208 naming interference sites (with their minimum positive thresholds) was $4.46 \mathrm{~mA}$ (range 1.5-9 mA, SD $1.44 \mathrm{~mA}$ ) (Fig. 3 left). Overall, 28 sites (13.5\%) were detected using an intensity 

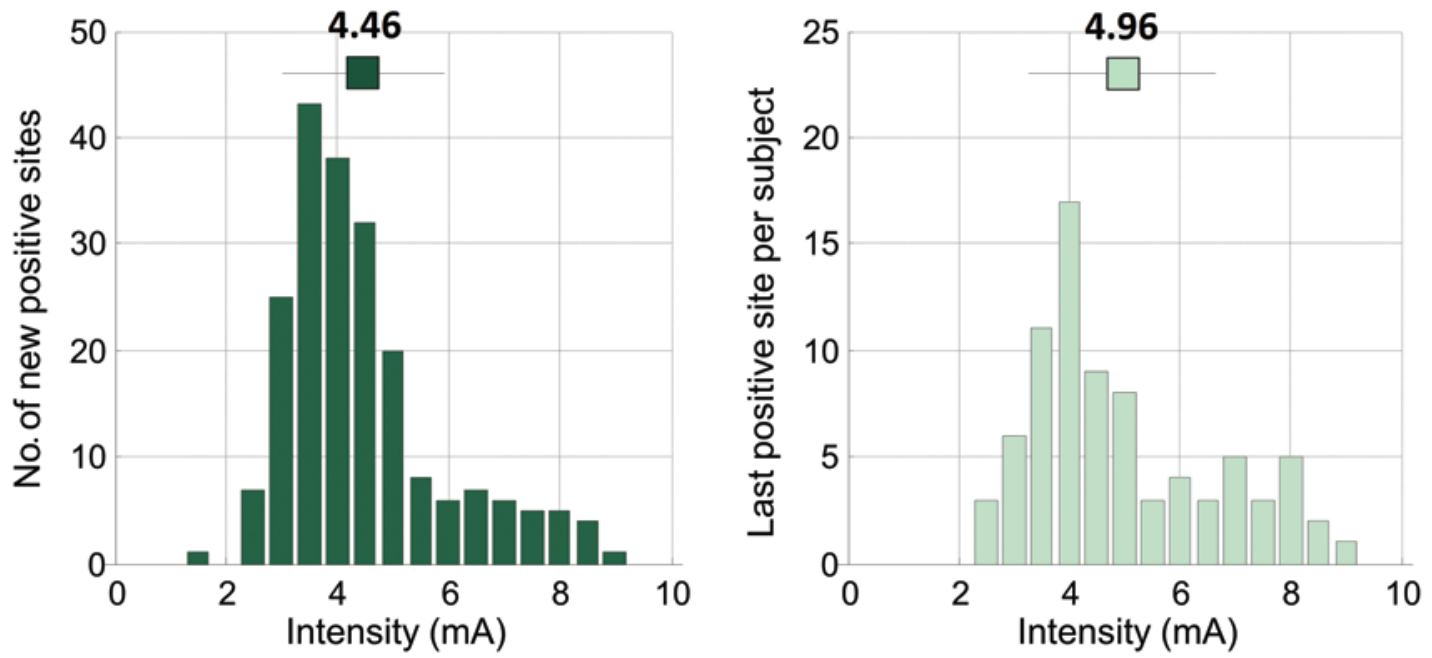

FIG. 3. Illustration of the intensities required to obtain a language interference. Left: Distribution of minimum intensities required to induce an interference from positive sites (208 interferences in 80 patients with positive mapping). Right: Distribution of maximum intensity required to trigger all the positive sites in each of the 80 patients. Note that, for instance, in nearly $25 \%$ of the patients (19 of 80), the mapping would not have been complete if an intensity higher than $6 \mathrm{~mA}$ had not been used. The solid squares and horizontal lines in the upper part of the graphs indicate, respectively, the means and standard deviations of the distributions. Figure is available in color online only.

of stimulation above $6 \mathrm{~mA}$. When we studied the higher stimulation intensity required to obtain all interferences in a given patient, we noted a mean intensity of $4.96 \mathrm{~mA}$ (range $2.5-9 \mathrm{~mA}, \mathrm{SD} 1.69 \mathrm{~mA}$ ) (Fig. 3 right). The minimum positive activation threshold did not differ significantly between female and male patients $(p=0.055)$ or between patients less than 50 years old and those more than 50 years old $(p=0.98)$. The interferences appeared as an "on/off" effect in 191 cases (for instance, no interference at $4 \mathrm{~mA}$ and anomia at $4.5 \mathrm{~mA}$ ). In 17 cases (9\%), the response to stimulation increments of $0.5 \mathrm{~mA}$ was less clear-cut, with delays, hesitations, and wondering about the type of response.

\section{Does the Level of Intensity Differ According to the Location of the Interference?}

A 1-way analysis of variance revealed a very significant effect of the cortical location on the minimum level of intensity required to produce a naming interference (1-way ANOVA df(3,167), $\left.F=16.58, p=1.78 \times 10^{-9}\right)$. Post hoc t-tests indicated that there was no statistically significant difference in the level of intensity for Broca's area (inferior frontal gyrus), the posterior temporal region, and the supramarginal gyrus (Fig. 4), but the main intensity used in the middle and superior frontal gyri was significantly higher than the main intensity of stimulation used in the other 3 regions ( $\mathrm{p}<0.0001$ for all 3 comparisons).

\section{Reproducibility of the Type of Response}

Each positive site was labeled with a type of interference (i.e., speech arrest, naming, phonological, semantic, or hesitation interferences) but repetitions of the same stimulation over the same site could modify the type of interference obtained. For instance, the first stimulation induced a naming interference at $5 \mathrm{~mA}$ and a second stimulation (a few minutes later), also at $5 \mathrm{~mA}$, induced a semantic interference. These variations of the type of interference over the brain mapping session with the same intensity of stimulation were observed at 13 sites $(6 \%)$ out of 208. In these cases, we classified the type of interference by performing more than 3 stimulations and labeling this site with the type of interference most frequently observed.

\section{Does the Type of Naming Interference at a Site Differ According to the Intensity of Stimulation?}

Increasing the intensity of stimulation on an interference site did not modify the type of interference observed in $168(81 \%)$ of 208 interferences. At 30 other interference sites $(14.5 \%)$, the type of interference was slightly modified. This was particularly the case for phonemic interferences (13 sites) where the interferences were more pronounced, with more syllables distorted. Some "hesitation" (12 sites) and semantic (5 sites) interferences were also more clearly detected (i.e., patients took longer to answer). Finally, in 10 interferences (4.5\%) (in 3 patients) the type of interference changed when the intensity of stimulation increased (always below the afterdischarge threshold). In all cases, anomia interferences found with the minimum positive threshold clearly became speech arrest interferences when the current intensity increased.

\section{Can We Accurately Discern the Cortical Extent of These Interferences?}

When detected with their minimum positive thresholds, the cortical extent of the positive sites varied from 9 to 120 $\mathrm{mm}^{2}$ with a mean area of approximately $20 \mathrm{~mm}^{2}$ (mean $19.74 \mathrm{~mm}^{2}$, SD 10.71). Fig. 5 illustrates the cortical extent of the interference sites detected.

The area of each interference site was evaluated by using the minimum positive threshold intensity and stimulating all the cortex located in the immediate vicinity. An increase in the stimulation intensity (below the "afterdis- 


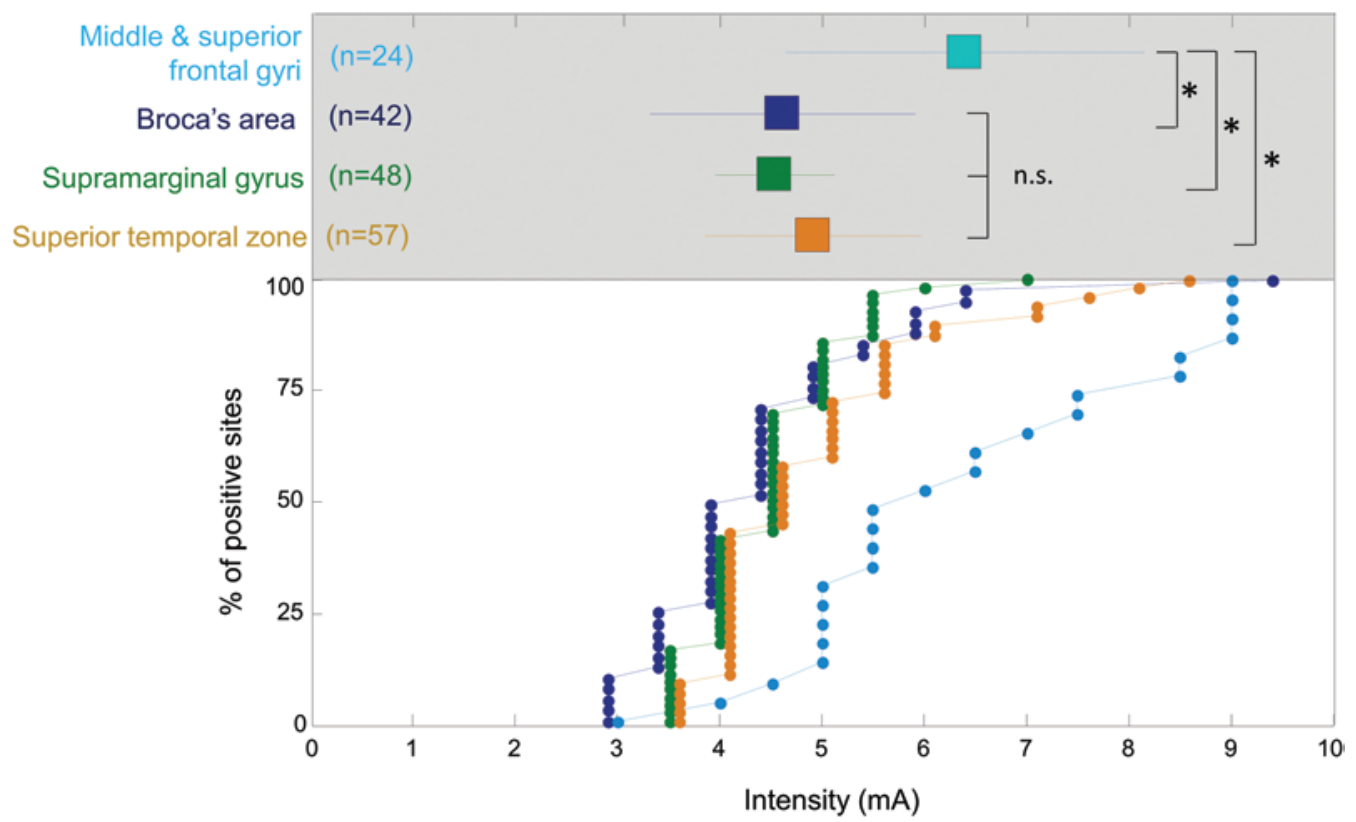

FIG. 4. Illustration of the intensity used according to some selected cortical regions. The lower part of the graph shows the distributions of intensities necessary to obtain language interference for different regions of the brain. The solid squares and related horizontal lines in the upper part of the graph indicate the mean intensity values and standard deviations for the different regions. The mean level of intensity necessary to obtain language interference was higher in the middle and frontal gyri $(6.35 \pm 1.78 \mathrm{~mA})$ than in Broca's area $(4.48 \pm 1.23 \mathrm{~mA})$, the posterior temporal region $(4.46 \pm 0.73 \mathrm{~mA})$, or the supramarginal gyrus $(4.81 \pm 1.19$ $\mathrm{mA}$ ). The "posterior temporal region" corresponds to the posterior part of the superior and middle temporal gyri (Regions 11,12 , and 13 of our classification scheme as illustrated in Fig. 1). *Statistically significant. n.s. = nonsignificant. Statistical significance is based on Student t-tests applied to differences in mean intensity. Figure is available in color online only.

charge threshold") led to an increase in the area of the positive sites in $94(45 \%)$ of 208 areas (i.e., stimulation of the cortex in the immediate vicinity of these areas resulted in new interference when the current increased). Overall, this phenomenon was found in 43 of the 80 positive mappings.

\section{Does the Level of Intensity Differ According to the Type of Interference Observed?}

As shown in Fig. 6, we found no significant relation between the type of interference observed and the minimum positive threshold intensity evoking that interference (1way ANOVA, $\mathrm{df}(4,202), \mathrm{F}=1.54, \mathrm{p}=0.19)$.

\section{Patients With No Response at $10 \mathrm{~mA}$ : Localization of the Areas Stimulated}

There were 20 completely negative brain mapping sessions involving various hemispheric regions-for instance, the inferior frontal gyrus (Broca's area), with 6 negative mappings for 98 stimulated sites; or the posterior temporal area, with 6 negative brain mappings for 84 stimulated sites. Other completely negative mapping sessions involved the middle and frontal gyri (130 sites tested) and upper parietal region (66 sites).

\section{Discussion}

Of the numerous publications on the topic, only a few have questioned the variability of brain mapping results according to the different electrostimulation parameters used. ${ }^{8,11,26,36,38}$ Other parameters being constant, only the intensity of stimulation for language can vary among authors, from those who did not stimulate the brain above 5 $\mathrm{mA},,^{20} 6 \mathrm{~mA}, 3,6,9,32$ or $10 \mathrm{~mA}^{18}$ to those who used intensities as high as $12 \mathrm{~mA},{ }^{36} 15 \mathrm{~mA},{ }^{37}$ or $16 \mathrm{~mA} .{ }^{25,26}$ Some influential authors did not use electrocorticography ${ }^{2}$ or found that a single current could often be selected for mapping, a current that just straddled the "afterdischarge threshold" in the left or right hemispheres. ${ }^{3}$ However, in rather small series, it has been shown that essential language areas could be detected at different intensities during extraoperative ${ }^{11}$ or intraoperative mapping. ${ }^{26}$ Furthermore, experimentation on macaques demonstrated that there was a graded increase in the area of activation when the stimulation current was increased. ${ }^{8}$ Finally, many debates have focused on the brain organization according to the type of response (for instance phonologic versus semantic response) found during electrostimulation brain mapping. ${ }^{4}$ Demonstrating that the type of response obtained may differ according to the level of stimulation could be not only clinically relevant but also of scientific value.

In this clinical trial with direct consequences for patient management, the intention was to focus on what could be considered as a main parameter for brain mapping-the intensity of stimulation. This parameter has rarely been studied intraoperatively in a prospective study involving a large cohort of patients. This study was not designed to test the effect of different frequencies (for instance $30 \mathrm{vs} 60 \mathrm{~Hz}$ ), as has been done in other studies, ${ }^{38}$ or durations of stimulation on patients. Our results can be summarized in 5 points. First, we found that, although a large majority of essential 


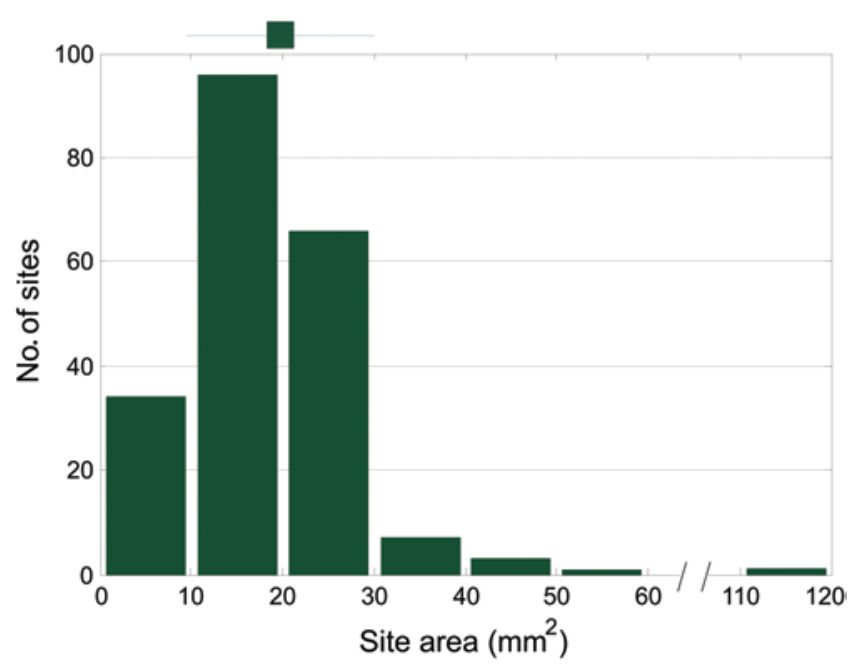

FIG. 5. Extent of the naming sites found. Naming areas were located in very small patches of cortex and were often separated by cortex unrelated to language. It was always surprising to observe that the displacement of the bipolar electrode on the cortex very close to a language area often induced no interference. Overall, 134 interference sites (60\%) were localized on cortical patches having a surface area of $20 \mathrm{~mm}^{2}$ or less. The solid square and the horizontal lines indicate the mean site area and its standard deviation. Figure is available in color online only.

language areas could be found by electrostimulation with a mean intensity of $4.46 \mathrm{~mA}$, a significant number of language areas $(13.5 \%)$ were detected with intensities above 6 $\mathrm{mA}-\mathrm{a}$ threshold considered in many publications as the maximum level of stimulation. ${ }^{32}$ This mean intensity of stimulation was higher than those found by some authors ${ }^{2}$ but quite similar to the findings of others. ${ }^{36}$ Thus, the positive minimum thresholds of essential areas varied among patients (from 1.5 to $9 \mathrm{~mA}$ ) but also within individual patients for different language areas (in $22 \%$ of cases). Second, stimulation of the same naming area with different intensities did not modify the type of response overall in the vast majority of cases $(81 \%)$. Nevertheless, in other cases, slight changes in the type of response or different types of responses (mainly transforming anomia into speech arrest) were detected. Third, essential areas detected were located in very small, subcentimeter cortical areas $(25 \%$ of the areas detected measured between 9 and $12 \mathrm{~mm}^{2}$ and $50 \%$ were smaller than $20 \mathrm{~mm}^{2}$ ). But this extent varied with the intensity of stimulation. Fourth, slightly higher thresholds of stimulation were observed in the left middle and superior frontal gyri compared with the inferior frontal and posterior temporal gyri. Finally, it has been shown experimentally that bipolar stimulation is able to reproduce similar activation patterns with the same stimulation parameters. ${ }^{8}$ This experimental finding was also observed in this clinical study: stimulation, with the same parameters, of the same cortical area across the brain mapping session reproduced the same type of interference in most cases. In some cases, we detected some variations in the type of response at some sites. It has already been shown by Lesser et al. ${ }^{10}$ that responses might occur at a given location during one trial but not in the next. However, the variability of the type of positive response to electrostimulation has rarely been described in the literature. Nevertheless, over

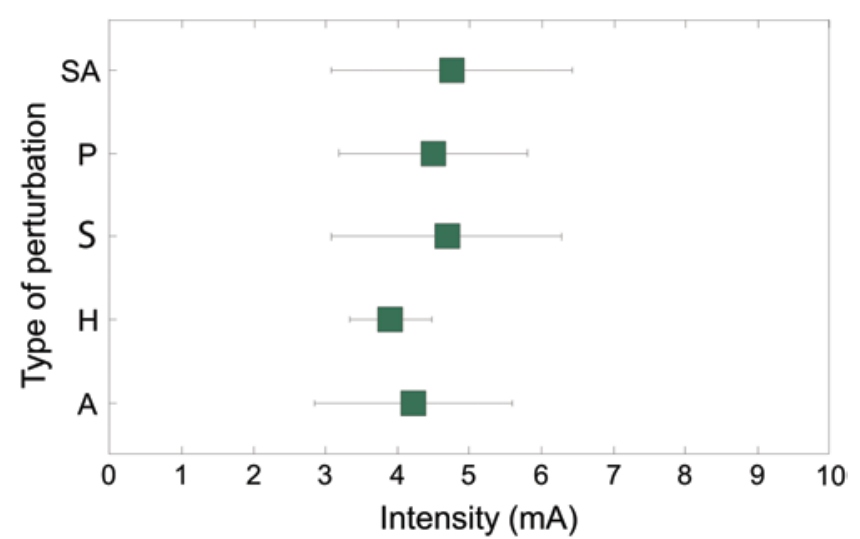

FIG. 6. Illustration of the intensity used according to the type of interference found. Although the mean intensity required to obtain a speech arrest was slightly higher than those required for other interferences, no significant relation was found between the type of interference and the current intensity. $A=$ anomia; $H=$ hesitation interference; $P=$ phonemic interference; $\mathrm{S}$ = semantic interference; $\mathrm{SA}=$ speech arrest. Figure is available in color online only.

these 100 mapping sessions, this response variability was not frequent ( $6 \%$ of the interferences detected).

\section{Intensity of Stimulation and Afterdischarges}

Overall, essential language sites were detected in most cases through an "on/off" effect. Similar threshold effects on naming tasks were also observed with repetitive transcranial magnetic stimulation. ${ }^{34}$ But graded responses were sometimes detected ( $9 \%$ of the cases) when varying the stimulation threshold by 0.5 or $1 \mathrm{~mA}$. In these cases, language function could just be perturbed (by a subthreshold effect) rather than inhibited.$^{27}$ Beyond this specific finding, in 10 sites $(4.5 \%)$ a marked increase in the level of stimulation intensity (for instance, when language areas detected at $4.5 \mathrm{~mA}$ were stimulated at $7 \mathrm{~mA}$ ) completely changed the type of response obtained. We hypothesize that this might have been due to recruitment of a distant motor area, the existence of areas with graded responses to stimulation, or the existence of an undetected stimulation bias.

Larger currents may generate local afterdischarges, which may spread to distant areas. ${ }^{35}$ Afterdischarges can be sources of mislocalization in language brain mapping. In this study, we detected afterdischarges in $9 \%$ of cases, a result in line with the findings of Blume et al., who detected afterdischarges in $8 \%$ of their stimulations. ${ }^{1}$ Various interpretations could be put forward for this finding. The authors who argue against the systematic use of electrocorticography ${ }^{2}$ would consider that this number is rather low and that 8 of our 9 brain mappings had afterdischarges detected with stimulation above $8 \mathrm{~mA}$ (i.e., stimulating below these intensities would avoid most afterdischarges). However, other authors found afterdischarges during electrostimulation mappings with lower intensities, such as a mean current intensity of $5.7 \mathrm{~mA}$ in the study by Zangaladze et al. ${ }^{38}$ Furthermore, if stimulation above $7.5 \mathrm{~mA}$ had been avoided in the present series, $5 \%$ of the essential language sites (10 of 208) would have been missed. Finally, the main issue could be: should brain mappings be performed using intensities just under the "afterdischarge 
threshold" or is it desirable to detect the minimum positive threshold of essential areas? These 2 thresholds are not always identical (the "afterdischarge threshold" is often higher than the minimum positive threshold). This study showed that, for some brain mapping sessions, varying the intensity did not change anything. Nevertheless, for a substantial number of patients, the number and extent of the areas detected and the type of response could vary. The use of different intensities of stimulation seemed essential to optimize brain mapping.

\section{Frontal Versus Temporal Differences and Extent of the Areas}

The mean stimulation threshold could be different in the frontal and temporal lobes. In their retrospective study of 39 patients, Wang et al. ${ }^{36}$ found a mean mapping threshold of $4.91 \mathrm{~mA}$ (a finding close to that of the present study) with an estimated mean stimulus threshold for the temporal language cortex 1.45 times higher than that for the frontal cortex. A similar frontal versus temporal difference was observed by Pouratian et al. ${ }^{26}$ in their 17 patients. We did not use the same definitions of "frontal" and "temporal" as these previous teams. This could explain why we detected no significant difference between Broca's area and the posterior temporal and supramarginal gyri (although the mean positive intensity was lower in Broca's area). Considering these results as a whole, we hypothesized that left hemispheric regions directly involved in language (such as inferior frontal, posterior temporal and supramarginal gyri) could globally have rather lower stimulation thresholds than other left hemispheric regions that are presumably less involved in language (such as the middle and superior frontal gyri or the upper parietal region).

Ojemann et al. ${ }^{18}$ stated that stimulated areas had "sharp boundaries" and "the estimated total surface area of essential sites was rather small, $2.5 \mathrm{~cm}^{2}$ or less in $50 \%$ and exceeding $6 \mathrm{~cm}^{2}$ in only $16 \%$ of the 117 subjects." The results reported here are in line with these findings and refine them. Our systematic measurements on each site revealed that cortical essential language sites were very discrete and slightly smaller than measured by Ojemann et al.: $50 \%$ of the detected sites had areas of less than $20 \mathrm{~mm}^{2}$. This very localized effect of bipolar stimulation is a clinical confirmation of the experimental findings of Haglund and others. ${ }^{8,15}$ Since detected areas can be very small, this could imply a need, in practice, to test more cortical areas by electrostimulation than previously advocated.

\section{Negative Brain Mapping Sessions}

Finally, many authors have pointed out that in a significant number of patients no essential language areas are detected in brain areas presumed to be involved in language. ${ }^{18,32}$ This was also the case in the present study. The reasons are probably multifactorial. In left hemispheric regions supposedly less involved in language (such as the middle or superior frontal gyri or anterior temporal regions), it was not surprising to find negative brain mapping. But variability was already frequent across the entire superior temporal gyrus in the landmark study of Ojemann et al., ${ }^{18}$ in which only $66 \%$ of the patients had a site where stimulation interfered with naming, or in Broca's area, where $20 \%$ of the stimulations were negative. Were these negative brain mapping sessions "really" negative (no naming area in the exposed cortex) or were they false negatives? According to some authors, certain patients showed reorganization of their language in these language regions, and tumor removal could be performed without language damage. ${ }^{6,12}$ But to err on the side of caution, it is worth remembering that negative brain mapping does not guarantee the absence of eloquent sites ${ }^{32}$ and naming should not be the sole task used to test language.

Although considered as the gold standard in many neurosurgical publications, electrostimulation brain mapping is not without drawbacks. Among other factors, some sources of false negatives during naming brain mapping sessions ${ }^{14}$ could be stimulation during the refractory period, inappropriate parameters of stimulation, or localization of the essential language sites in the sulci. This last hypothesis has very rarely been considered, ${ }^{19}$ but every neurosurgeon knows that it is difficult to stimulate within sulci. There is a lack of data regarding the possible location of essential areas within the sulci, and we hypothesize that naming areas may also be located there but could have remained undetected. This study also showed that subthreshold stimulations could be a potential source of false negatives. Another such source in our study could be the fact that stimulation did not exceed $10 \mathrm{~mA}$. It is possible that essential language areas would have been detected above this threshold as evoked by other authors. ${ }^{26}$

\section{Conclusions}

This prospective study showed that significant variations in the number of interferences, the type of interference detected, and their cortical extent were observed when the intensity of stimulation was increased. Considering these findings, we think that electrostimulation for cortical brain mapping should be performed using different intensities of stimulation. This could refine the clinical results and scientific data derived from brain mapping.

\section{Acknowledgments}

We thank Eve Salleles, Lucile Mazières, and Susan Becker for editorial assistance.

\section{References}

1. Blume WT, Jones DC, Pathak P: Properties of after-discharges from cortical electrical stimulation in focal epilepsies. Clin Neurophysiol 115:982-989, 2004

2. Boetto J, Bertram L, Moulinié G, Herbet G, Moritz-Gasser S, Duffau H: Low rate of intraoperative seizures during awake craniotomy in a prospective cohort with 374 supratentorial brain lesions: electrocorticography is not mandatory. World Neurosurg 84:1838-1844, 2015

3. Chang EF, Wang DD, Perry DW, Barbaro NM, Berger MS: Homotopic organization of essential language sites in right and bilateral cerebral hemispheric dominance. J Neurosurg 114:893-902, 2011

4. Corina DP, Loudermilk BC, Detwiler L, Martin RF, Brinkley JF, Ojemann G: Analysis of naming errors during cortical stimulation mapping: implications for models of language representation. Brain Lang 115:101-112, 2010 
5. Deloche GH, Hannequin D: Test de dénomination orale de 80 images: DO 80. Paris: ECPA, 1997

6. Duffau H: Awake mapping and tumor surgery, in Duffau $\mathrm{H}$ (ed): Brain Mapping, from Neural Basis of Cognition to Surgical Applications. Vienna: Springer, 2011

7. Gallentine WB, Mikati MA: Intraoperative electrocorticography and cortical stimulation in children. J Clin Neurophysiol 26:95-108, 2009

8. Haglund MM, Ojemann GA, Blasdel GG: Optical imaging of bipolar cortical stimulation. J Neurosurg 78:785-793, 1993

9. Kim SS, McCutcheon IE, Suki D, Weinberg JS, Sawaya R, Lang FF, et al: Awake craniotomy for brain tumors near eloquent cortex: correlation of intraoperative cortical mapping with neurological outcomes in 309 consecutive patients. Neurosurgery 64:836-845, 345-346, 2009

10. Lesser RP, Lee HW, Webber WR, Prince B, Crone NE, Miglioretti DL: Short-term variations in response distribution to cortical stimulation. Brain 131:1528-1539, 2008

11. Lesser RP, Lüders H, Klem G, Dinner DS, Morris HH, Hahn J: Cortical afterdischarge and functional response thresholds: results of extraoperative testing. Epilepsia 25:615-621, 1984

12. Lubrano V, Draper L, Roux FE: What makes surgical tumor resection feasible in Broca's area? Insights into intraoperative brain mapping. Neurosurgery 66:868-875, 2010

13. Lüders H, Lesser RP, Hahn J, Dinner DS, Morris H, Resor S, et al: Basal temporal language area demonstrated by electrical stimulation. Neurology 36:505-510, 1986

14. Mandonnet E, Winkler PA, Duffau H: Direct electrical stimulation as an input gate into brain functional networks: principles, advantages and limitations. Acta Neurochir (Wien) 152:185-193, 2010

15. Nathan SS, Sinha SR, Gordon B, Lesser RP, Thakor NV: Determination of current density distributions generated by electrical stimulation of the human cerebral cortex. Electroencephalogr Clin Neurophysiol 86:183-192, 1993

16. Nespoulous JL, Joanette Y, Lecours AR: MT 86: examen linguistique du sujet aphasique. Isbergues, France: L'Ortho-Edition, 1992

17. Ojemann G: Functional mapping of cortical language areas in adults, in Devinsky O, Berić A, Dogali M (eds): Electrical and Magnetic Stimulation of the Brain and Spinal Cord. New York: Raven, 1993

18. Ojemann G, Ojemann J, Lettich E, Berger M: Cortical language localization in left, dominant hemisphere. An electrical stimulation mapping investigation in 117 patients. J Neurosurg 71:316-326, 1989

19. Ojemann GA: Cortical organization of language. J Neurosci 11:2281-2287, 1991

20. Ojemann JG, Miller JW, Silbergeld DL: Preserved function in brain invaded by tumor. Neurosurgery 39:253-259, 1996

21. Ojemann SG, Berger MS, Lettich E, Ojemann GA: Localization of language function in children: results of electrical stimulation mapping. J Neurosurg 98:465-470, 2003

22. Oldfield RC: The assessment and analysis of handedness: the Edinburgh inventory. Neuropsychologia 9:97-113, 1971

23. Penfield W, Rasmussen T: The Cerebral Cortex of Man. A Clinical Study of Localization of Function. New York: Hafner, 1954

24. Penfield W, Robert L: Speech and Brain Mechanisms. Princeton, NJ: Princeton University Press, 1959

25. Pouratian N, Bookheimer SY, Rex DE, Martin NA, Toga AW: Utility of preoperative functional magnetic resonance imaging for identifying language cortices in patients with vascular malformations. J Neurosurg 97:21-32, 2002

26. Pouratian N, Cannestra AF, Bookheimer SY, Martin NA, Toga AW: Variability of intraoperative electrocortical stimu- lation mapping parameters across and within individuals. J Neurosurg 101:458-466, 2004

27. Ranck JB Jr: Which elements are excited in electrical stimulation of mammalian central nervous system: a review. Brain Res 98:417-440, 1975

28. Riva M, Fava E, Gallucci M, Comi A, Casarotti A: Monopolar high-frequency language mapping: can it help in the surgical management of gliomas? A comparative clinical study. J Neurosurg 25:1-11, 2015

29. Roux FE, Lubrano V, Lauwers-Cances V, Trémoulet M, Mascott CR, Démonet JF: Intra-operative mapping of cortical areas involved in reading in mono- and bilingual patients. Brain 127:1796-1810, 2004

30. Roux FE, Miskin K, Durand JB, Sacko O, Réhault E, Tanova $\mathrm{R}$, et al: Electrostimulation mapping of comprehension of auditory and visual words. Cortex 71:398-408, 2015

31. Sacko O, Lauwers-Cances V, Brauge D, Sesay M, Brenner A, Roux FE: Awake craniotomy vs surgery under general anesthesia for resection of supratentorial lesions. Neurosurgery 68:1192-1199, 2011

32. Sanai N, Berger MS: Mapping the horizon: techniques to optimize tumor resection before and during surgery. Clin Neurosurg 55:14-19, 2008

33. Snodgrass JG, Vanderwart M: A standardized set of 260 pictures: norms for name agreement, image agreement, familiarity, and visual complexity. J Exp Psychol Hum Learn 6:174-215, 1980

34. Sparing R, Mottaghy FM, Hungs M, Brügmann M, Foltys H, Huber W, et al: Repetitive transcranial magnetic stimulation effects on language function depend on the stimulation parameters. J Clin Neurophysiol 18:326-330, 2001

35. Van Buren JM, Lewis DV, Schuette WH, Whitehouse WC, Marsan CA: Fluorometric monitoring of NADH levels in cerebral cortex: preliminary observations in human epilepsy. Neurosurgery 2:114-121, 1978

36. Wang SG, Eskandar EN, Kilbride R, Chiappa KH, Curry WT, Williams Z, et al: The variability of stimulus thresholds in electrophysiologic cortical language mapping. J Clin Neurophysiol 28:210-216, 2011

37. Winkler PA: Extraoperative electrical mapping, in Duffau $\mathrm{H}$ (ed): Brain Mapping, from Neural Basis of Cognition to Surgical Applications. Vienna: Springer, 2011

38. Zangaladze A, Sharan A, Evans J, Wyeth DH, Wyeth EG, Tracy JI, et al: The effectiveness of low-frequency stimulation for mapping cortical function. Epilepsia 49:481-487, 2008

\section{Disclosures}

The authors report no conflict of interest concerning the materials or methods used in this study or the findings specified in this paper.

\section{Author Contributions}

Conception and design: Roux. Acquisition of data: Roux. Analysis and interpretation of data: Roux, Durand, Djidjeli. Drafting the article: Roux, Djidjeli. Critically revising the article: Durand, Djidjeli, Giussani. Statistical analysis: Durand. Administrative/ technical/material support: Djidjeli, Moyse. Study supervision: Giussani.

\section{Correspondence}

Franck-Emmanuel Roux, Service de Neurochirurgie et CNRS (CERCO), Hôpital Pierre-Paul Riquet, F-31059 Toulouse, France. email: franck_emmanuel.roux@yahoo.fr. 